\title{
A UNIQUE DECOMPOSITION THEOREM FOR 3-MANIFOLDS WITH CONNECTED BOUNDARY
}

\author{
BY \\ JONATHAN L. GROSS( $\left.{ }^{1}\right)$
}

1. Introduction. The concern of this paper is the class of triangulated, orientable, connected compact 3-manifolds with connected nonvacuous boundary. If $M$ and $M^{\prime}$ are two manifolds in this class, one forms their disk sum $M \triangle M^{\prime}$ by pasting a 2-cell on bd $(M)$ to a 2-cell on bd $\left(M^{\prime}\right)$. Up to homeomorphism, the operation of disk sum is well defined, associative, and commutative. One says that a manifold $P$ in this class is $\triangle$-prime if $P$ is not a 3-cell, and whenever $P$ is homeomorphic to a disk sum $M \triangle M^{\prime}$, either $M$ or $M^{\prime}$ is a 3-cell.

The goal of this paper is the proof of the following theorem, which answers a question of Milnor [3, p. 6] in the affirmative:

DECOMPOSITION THEOREM. Let $M$ be a triangulated, orientable, connected compact 3-manifold with connected nonvacuous boundary. If $M$ is not a 3-cell, then $M$ is homeomorphic to a disk sum $P_{1} \triangle \cdots \triangle P_{n}$ of $\triangle$-prime 3-manifolds. The summands $P_{i}$ are uniquely determined up to order and homeomorphism.

It will be assumed from now on that any manifold is triangulated, orientable, connected and compact. Any map considered is piecewise linear. It will occasionally be convenient to refer to the 3-cell (which is an identity element for the disk sum) or the 3-sphere (which is an identity element for the connected sum-see below) as a trivial 3-manifold.

The connected sum $M \# M^{\prime}$ of two 3-manifolds is obtained by removing from each the interior of a 3-cell and then pasting the resulting boundary components together. Up to homeomorphism, the operation of connected sum is well defined, associative, and commutative. One says that a 3-manifold $P$ is \#-prime if $P$ is not a 3-sphere, and whenever $P$ is homeomorphic to a connected sum $M \# M^{\prime}$, either $M$ or $M^{\prime}$ is a 3-sphere.

Definition. Let $P C C$ denote the class of 3-manifolds $M$ with connected nonvacuous boundary such that every 2 -sphere in $M$ bounds a 3-cell.

Most of the work of this paper is in proving that the Decomposition Theorem holds for 3-manifolds in the class $P C C$, which will be done in $\$ 3$. The lemmas of

Presented to the Society, January 24, 1968; received by the editors April 17, 1968 and, in revised form, November 18, 1968.

(1) The author wishes to thank Professor Edward M. Brown, under whose guidance this work was done. This paper was written at Dartmouth College and was partially supported by National Science Foundation Grant GP6526. 
$\$ 2$ provide major assistance for that proof. The extension of the Decomposition Theorem from 3-manifolds in PCC to all 3-manifolds with connected nonvacuous boundary will be accomplished in $\$ 4$ by an application of Theorems 1 and 2 below. One observes the analogy between the Decomposition Theorem and Theorem 2.

THEOREM 1. Let $M$ be a 3-manifold with connected nonvacuous boundary. Then $M$ is homeomorphic to a connected sum of a (possibly trivial) 3-manifold in PCC and a (possibly trivial) 3-manifold with vacuous boundary. The summands are uniquely determined up to homeomorphism.

The proof of Theorem 1 follows readily from remark 1 of Milnor [3, p. 5].

THEOREM 2. Let $M$ be a nontrivial 3-manifold with vacuous boundary. Then $M$ is homeomorphic to a connected sum $P_{1} \# \cdots \# P_{n}$ of \#-prime 3-manifolds. The summands $P_{i}$ are uniquely determined up to order and homeomorphism.

The present Theorem 2 is Theorem 1 of Milnor [3].

Extension of results. The author uses the methods of this paper to show in [1] that there is a unique decomposition theorem for compact, orientable 3-manifolds with several boundary components. It is also shown in [1] that the problem of classifying the compact, orientable 3-manifolds with several boundary components reduces to the problem of classifying the $\triangle$-prime 3-manifolds.

2. Some lemmas for Theorem 3. Let $R$ be a 2-manifold of genus zero whose boundary has $n+1$ components, for some nonnegative integer $n$, i.e. $R$ is a disk with $n$ holes. If a 3-manifold $M$ is homeomorphic to $R \times[0,1]$, then $M$ is called a handlebody of genus $n$. In the case that $n=0$, one says $M$ is a trivial handlebody. In the case that $n=1$, one say that $M$ is a handle.

LEMMA 1. Let $T$ be the union of a finite family of handlebodies $K_{1}, \ldots, K_{n}$ (some of which, perhaps, are trivial) such that the intersection of any $K_{j}$ with the union of all the other handlebodies $K_{i}$ is a finite union of disjoint disks on bd $\left(K_{j}\right)$. If T is orientable and connected, then $T$ is a (possibly trivial) handlebody.

The proof of Lemma 1, which is omitted here, is by an induction on the number $n$.

Lemma 2. Let $M$ be a 3-manifold and let $D$ be a disk in bd (M). Then there is a 3-cell $K$ in $M$, lying in an arbitrarily small neighborhood of $D$, such that $K \cap$ bd $(M)$ =D. And $M \approx \operatorname{cl}(M-K)$.

The proof of Lemma 2 is omitted.

LEMMA 3. Let $Y$ be the union of a finite collection of disjoint 3-manifolds and let $T$ be the union of a finite collection of disjoint handlebodies, such that $Y$ and $T$ intersect only on their boundaries, such that the intersection of bd $(T)$ with each component of $\mathrm{bd}(Y)$ is the union of a finite nonvoid family of disjoint disks, and such that $Y \cup T$ is orientable and connected. Then $Y$ contains a family of 3-cells, whose union will be denoted by $K$, such that for $Y^{\prime}=\mathrm{cl}(Y-K)$ and $T^{\prime}=T \cup K$ the following conditions 
hold: (i) $Y^{\prime} \approx Y$. (ii) $T^{\prime}$ is the union of a finite collection of disjoint handlebodies. (iii) $Y^{\prime}$ and $T^{\prime}$ intersect only on their boundaries. (iv) The intersection of bd $\left(T^{\prime}\right)$ with each component of bd $\left(Y^{\prime}\right)$ is a single disk.

Proof. For each component $R_{j}$ of bd $(Y)$, let $D_{j}$ be a disk which contains bd $(T) \cap R_{j}$ in its interior and let $K_{j}$ be a 3-cell in $Y$ such that $K_{j} \cap R_{j}=D_{j}$, with the 3-cells $K_{j}$ chosen so that they are mutually disjoint (see Lemma 2). Let $K$ be the union of the 3-cells $K_{j}$. It follows from Lemmas 1 and 2 that conditions (i) and (ii) of the conclusion hold. Conditions (iii) and (iv) of the conclusion evidently follow from the construction.

Definition. Let $M$ and $N$ be manifolds, and let $f: M \rightarrow N$ be an imbedding. One says that $f$ is a proper imbedding if $f(\mathrm{bd}(M)) \subset \mathrm{bd}(N)$ and $f(\operatorname{int}(M)) \subset \operatorname{int}(N)$.

In Lemma 4 below and thereafter, a homeomorphism $(X, U) \rightarrow(Y, V)$ will indicate a homeomorphism of $X$ onto $Y$ which carries a subspace $U$ of $X$ onto a subspace $V$ of $Y$.

LEMma 4. Let $M$ be a 3-manifold in the class PCC. Let $D$ be a disk, properly imbedded in $M$. Let $E$ be another disk, also properly imbedded in $M$, such that $\mathrm{bd}(D) \cap \mathrm{bd}(E)$ is void and such that $\mathrm{bd}(D) \cup \mathrm{bd}(E)$ is the boundary of an annulus on bd $(M)$. Then there is a homeomorphism $(M, D) \rightarrow(M, E)$.

The proof of Lemma 4, which is omitted here, is by a general position argument to reduce the intersection of the disks to a family of simple loops and an induction argument involving cutting at an innermost intersection loop on one of the disks.

LemMA 5. Let $Y$ and $Y^{\prime}$ be homeomorphic 3-manifolds with nonempty boundary, and let $C$ and $C^{\prime}$ be 3-cells such that $C \cap Y=\operatorname{bd}(C) \cap \operatorname{bd}(Y)$ and $C^{\prime} \cap Y^{\prime}$ $=\mathrm{bd}\left(C^{\prime}\right) \cap \mathrm{bd}\left(Y^{\prime}\right)$, and such that bd $(C)$ intersects each component of bd $(Y)$ in $a$ disk and bd $\left(C^{\prime}\right)$ intersects each component of bd $\left(Y^{\prime}\right)$ in a disk. If $Y \cup C$ and $Y^{\prime} \cup C^{\prime}$ are orientable, then $Y \cup C \approx Y^{\prime} \cup C^{\prime}$.

The proof of Lemma 5 is omitted.

LeMmA 6. Let $P$ be a $\triangle$-prime 3-manifold in the class $P C C$, and let $E_{1}, \ldots, E_{n}$ be a collection of disjoint disks, each properly imbedded in $P$. Let $T$ be the union of the closures of the components of $P-\left(E_{1} \cup \cdots \cup E_{n}\right)$ which are 3-cells, and let $Y=$ $\mathrm{cl}(P-T)$ be nonvoid. Let $C$ be a 3-cell such that $C$ and $Y$ intersect only on their boundaries, such that bd $(C)$ intersects each component of bd $(Y)$ in a disk, and such that $C \cup Y$ is orientable. Then $Y \cup C \approx P$.

Proof. By Lemma 1, each component of $T$ is a handlebody. Applying Lemma 3 to $Y$ and $T$, one obtains $K, Y^{\prime}$, and $T^{\prime}$ as in the conclusion of Lemma 3. The number of components of $T^{\prime}$ is the number of components of bd $(P)$, by condition (iv) of Lemma 3. Thus $T^{\prime}$ is a single handlebody. Choose a disk $D$ on bd $\left(T^{\prime}\right)$ such that $D$ contains $T^{\prime} \cap Y^{\prime}$ in its interior. By Lemma 2, there is a 3-cell $B$ in the handlebody $T^{\prime}$ such that $B \cap$ bd $\left(T^{\prime}\right)=D$. Since $Y$ is nonvoid, $Y^{\prime} \cup B$ is a 
nontrivial 3-manifold in PCC. One observes that $P=\left(Y^{\prime} \cup B\right) \triangle \mathrm{cl}\left(T^{\prime}-B\right)$. Since $T^{\prime} \approx \mathrm{cl}\left(T^{\prime}-B\right)$, since $P$ is $\triangle$-prime, and since $Y^{\prime} \cup B$ is nontrivial, the handlebody $T^{\prime}$ must be trivial. The conclusion now follows from Lemma 5 because $Y \approx Y^{\prime}$.

Lemma 7. Let $P$ be a $\triangle$-prime 3-manifold in the class $P C C$ and let $E_{1}, \ldots,{ }^{\circ} E_{n}$ be a collection of disjoint disks, each properly imbedded in $P$. (a) The closure in $P$ of all but possibly one component of $P-\left(E_{1} \cup \cdots \cup E_{n}\right)$ is a 3-cell. (b) If the closure in $P$ of every component of $P-\left(E_{1} \cup \cdots \cup E_{n}\right)$ is a 3-cell, then $P$ is a handle.

Proof of (a). Let $Y$ be the union of the closures of the components of $P$ $\left(E_{1} \cup \ldots \cup E_{n}\right)$ which are not 3-cells. Let $C$ be a 3-cell such that $C$ and $Y$ intersect only on their boundaries and bd $(C)$ intersects each component of bd $(Y)$ in a disk, and $Y \cup C$ is orientable. By Lemma 6, $Y \cup C \approx P$. One first considers the case in which $Y$ is not connected. In this case, let $D$ be a disk on bd $(C)$ which contains in its interior all of the components of the intersection of bd $(C)$ with some component of $Y$. By Lemma 2, there is a 3-cell $B$ in $C$ such that $B \cap$ bd $(C)$ $=D$. The disk $B \cap \mathrm{cl}(C-B)$ splits $Y \cup C$ into two nontrivial disk summands, which contradicts the hypothesis that $P$ is $\triangle$-prime. So the case that $Y$ is not connected cannot occur.

Now let $Y_{1}$ be the closure of a component of $P-\left(E_{1} \cup \cdots \cup E_{n}\right)$ which is not a 3-cell. If $Y \neq Y_{1}$, then $Y_{1} \cap \operatorname{cl}\left(Y-Y_{1}\right)$ is the union of a subset of the disks $E_{1}, \ldots, E_{n}$. Renumber the disks so that $Y_{1} \cap \operatorname{cl}\left(Y-Y_{1}\right)=E_{1} \cup \cdots \cup E_{r}$. For $j=1, \ldots, r$, let $D_{j}$ be a disk in bd $\left(Y_{1}\right)$ which contains $E_{j}$ in its interior, and let $B_{j}$ be a 3-cell in $Y_{1}$ such that $B_{j} \cup$ bd $\left(Y_{1}\right)=D_{j}$ and such that the 3-cells $B_{j}$ are mutually disjoint (see Lemma 2). For $j=1, \ldots, r$, let $E_{n+j}$, be the disk $B_{j} \cap \operatorname{cl}\left(Y-B_{j}\right)$. One observes that the disks $E_{1}, \ldots, E_{n+r}$ are disjoint and properly imbedded in $P$. One also observes that the union $Y^{\prime}$ of the closures of the components of $P$ $\left(E_{1} \cup \ldots \cup E_{n+r}\right)$ which are not 3-cells is not connected, for one of its components is $\mathrm{cl}\left(Y_{1}-\left(B_{1} \cup \ldots \cup B_{r}\right)\right)$ and the union of the rest of its components is $\mathrm{cl}\left(Y-Y_{1}\right)$. But as above, this contradicts the hypothesis that $P$ is $\triangle$-prime. Therefore $Y_{1}=Y$, which completes (a).

Proof of (b). Suppose that the closure in $P$ of every component of $P$ $\left(E_{1} \cup \cdots \cup E_{n}\right)$ is a 3-cell. The intersection of two of these 3-cells is the union of some of the disks $E_{j}$. Therefore, these 3-cells satisfy the criteria for the handlebodies of Lemma 1 . Hence, $P$ is a handlebody. Since $P$ is $\triangle$-prime, its genus is one.

3. Decomposition in PCC. This entire section is devoted to the proof of Theorem 3, which is the restriction of the Decomposition Theorem to 3-manifolds in the class $P C C$.

THEOREM 3. Let $M$ be a nontrivial 3-manifold in the class PCC. (a) Then $M$ is homeomorphic to a sum $P_{1} \triangle \cdots \triangle P_{n}$ of $\triangle$-prime 3-manifolds. (b) The summands $P_{i}$ are uniquely determined up to order and homeomorphism. 
Proof of (a). Suppose that $M$ is not already $\triangle$-prime. Then $M$ is homeomorphic to a disk sum $M_{1} \triangle M_{2}$ where $M_{1}$ and $M_{2}$ are both nontrivial. One observes that a 3-manifold in the class $P C C$ is bounded by a 2-sphere if and only if it is a 3-cell. Hence, the genera of bd $\left(M_{1}\right)$ and bd $\left(M_{2}\right)$ are positive. One also observes that genus $(\mathrm{bd}(M))=$ genus $\left(\mathrm{bd}\left(M_{1}\right)\right)+$ genus $\left(\mathrm{bd}\left(M_{2}\right)\right)$. Therefore, the decomposing process terminates in not more than genus (bd $(M)$ ) steps, which proves (a).

Proof of (b). Suppose that $M \approx P_{1} \triangle \cdots \triangle P_{n}$, where $P_{1}, \ldots, P_{n}$ are $\triangle$-prime 3-manifolds. Then let $K$ be a 3-cell, and let $D_{1}, \ldots, D_{n}$ be disjoint disks on bd $(K)$. The 3-manifold $M^{*}$ is obtained by pasting a disk on bd $\left(P_{j}\right)$ to $D_{j}$, for $j=1, \ldots, n$ (see Figure 1). Since $M^{*} \approx P_{1} \triangle \cdots \triangle P_{n}$, it follows that $M^{*}$ is homeomorphic to $M$. It will be convenient to work with $M^{*}$.

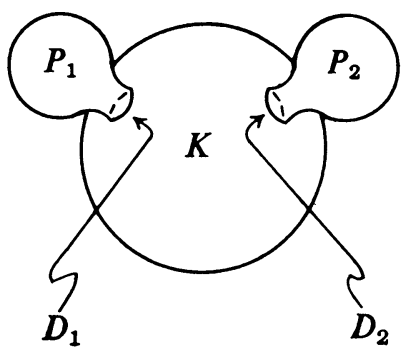

FIGURE 1

Let $E$ be a properly imbedded disk in $M^{*}$ such that $M^{*}-E$ has two components and such that the closure of each of these components is nontrivial. The closures of the components of $M^{*}-E$ will be called $M_{1}$ and $M_{2}$.

From an induction on the number $n$ of $\triangle$-prime summands $P_{i}$ of $M^{*}$, it follows that (b) of the theorem can be proved by establishing the following statement (which will be done):

A. There is a renumbering of the $P_{i}$ 's such that for some integer $r$ with $1 \leqq r \leqq n$, $M_{1} \approx P_{1} \triangle \cdots \triangle P_{r}$ and $M_{2} \approx P_{r+1} \triangle \cdots \triangle P_{n}$.

It will be assumed that the components of $E \cap\left(D_{1} \cup \ldots \cup D_{n}\right)$ are simple arcs and simple loops, each a crossing of surfaces, for a general position argument indicates that it suffices to consider this case.

Basis step. Suppose that $E \cap\left(D_{1} \cup \cdots \cup D_{n}\right)$ is void. One assumes that the disk $E$ lies in some $P_{j}$, for otherwise $E$ would lie in the 3-cell $K$, which would imply statement A immediately. Surely $P_{j}-E$ has two components, for otherwise $M^{*}-E$ would have but one component. Moreover, since $P_{j}$ is $\triangle$-prime, the closure $X$ of one of the two components of $P_{j}-E$ is a 3-cell. The disk $D_{j}$ lies on bd $(X)$, for otherwise $X$ would be the closure of a component of $M^{*}-E$, i.e. $X$ would be $M_{1}$ or $M_{2}$, contradicting their nontriviality. If $X \subset M_{1}$, then

$$
M_{2} \approx P_{j} \text { and } M_{1} \approx P_{1} \triangle \cdots \triangle P_{j-1} \triangle P_{j+1} \triangle \cdots \triangle P_{n} .
$$


Obviously, a similar decomposition results if $X \subset M_{2}$. Therefore, statement A holds.

The statements below numbered $(3.1), \ldots,(3.7)$ are important intermediate results in the proof of the induction step.

Induction step. Let $E \cap\left(D_{1} \cup \cdots \cup D_{n}\right)$ have exactly $m$ components.

(3.1) If any component of $E \cap\left(D_{1} \cup \cdots \cup D_{n}\right)$ is a loop, then statement A holds.

Proof of (3.1). If any component of $E \cap\left(D_{1} \cup \ldots \cup D_{n}\right)$ is a loop, then there is a component $k$ of $E \cap\left(D_{1} \cup \ldots \cup D_{n}\right)$ which is a loop and which bounds a subdisk $D^{\prime}$ of some $D_{j}$ such that $D^{\prime} \cap E=k$. Let $E^{\prime}$ be the subdisk of $E$ which $k$ bounds.

Let $E_{1}$ be a disk which lies near but does not intersect the disk $\left(E-E^{\prime}\right) \cup D^{\prime}$, which is properly imbedded in $M^{*}$, which lies in general position with respect to $D_{1} \cup \cdots \cup D_{n}$, and which meets $D_{1} \cup \cdots \cup D_{n}$ in fewer than $m$ loops (see Figure 2). By Lemma 4, there is a homeomorphism $f:\left(M^{*}, E\right) \rightarrow\left(M^{*}, E_{1}\right)$. Let $M_{1}^{\prime}=f\left(M_{1}\right)$ and $M_{2}^{\prime}=f\left(M_{2}\right)$. Since the number of components of $E_{1} \cap\left(D_{1} \cup \ldots\right.$ $\left.\cup D_{n}\right)$ is less than $m$, there is a renumbering of the $P_{i}$ 's such that $M_{1}^{\prime} \approx P_{1} \triangle \cdots \triangle P_{r}$ and $M_{2}^{\prime} \approx P_{r+1} \triangle \cdots \triangle P_{n}$. Hence, $M_{1} \approx P_{1} \triangle \cdots \triangle P_{r}$ and $M_{2} \approx P_{r+1} \triangle \cdots \triangle P_{n}$. Thus, statement (3.1) is proved.

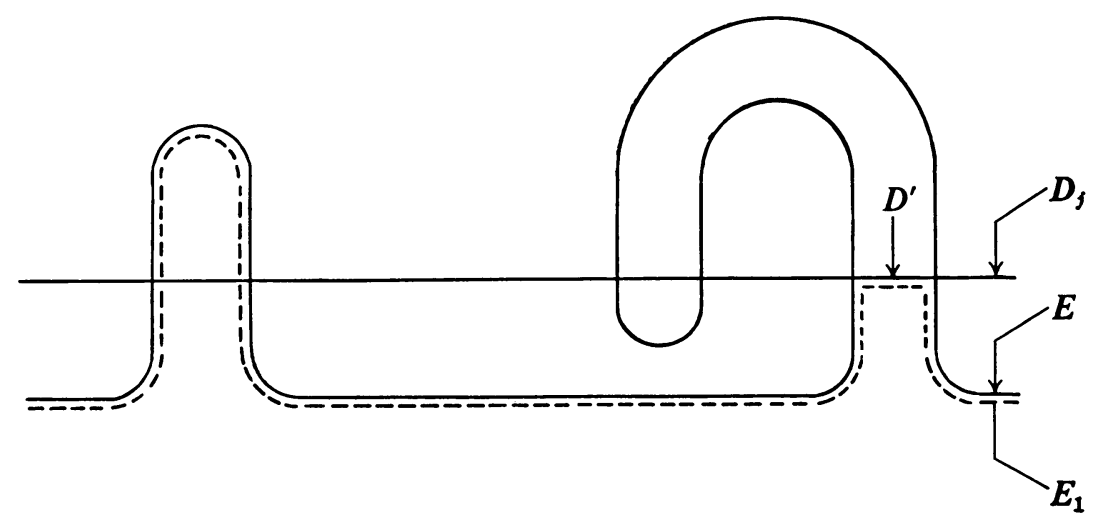

FIGURE 2

It will hereafter be assumed that every component of $E \cap\left(D_{1} \cup \cdots \cup D_{n}\right)$ is an arc. This implies, of course, that for $j=1, \ldots, n$, each component of the intersection of the disk $E$ with the $\triangle$-prime summand $P_{j}$ is a properly imbedded disk.

Definition. A $\triangle$-prime summand $P_{j}$ of $M^{*}$ is called a special handle if the closure of every component of $P_{j}-E$ is a 3-cell. (It follows from (b) of Lemma 7 that a special handle actually is a handle.)

Definition. Let $P_{j}$ be a $\triangle$-prime summand of $M^{*}$ such that $P_{j}$ is not a special handle. The essence of $P_{j}$, hereafter denoted by $Y_{j}$, is the closure in $P_{j}$ of the 
component of $P_{j}-E$ which is not a 3-cell. (It follows from (a) of Lemma 7 that $Y_{j}$ is well defined.)

One renumbers $P_{1}, \ldots, P_{n}$ so that $P_{1}, \ldots, P_{u}$ are the summands whose essences lie in $M_{1}$, that $P_{u+1}, \ldots, P_{s}$ are the summands whose essences lie in $M_{2}$, and that $P_{s+1}, \ldots, P_{n}$ are the special handles.

(3.2) Each component of $\operatorname{cl}\left(M_{1}-\left(Y_{1} \cup \cdots \cup Y_{u}\right)\right)$ is a (possibly trivial) handlebody.

Proof of (3.2). A component of $\mathrm{cl}\left(M_{1}-\left(Y_{1} \cup \ldots \cup Y_{u}\right)\right)$ is the union of 3-cells, each of which is either the closure of a component of $K-E$ or the closure of a component of some $P_{j}-E$. Each component of the intersection of any two of these 3-cells is the closure of a component of some $D_{i}-E$. Therefore, each component of $\mathrm{cl}\left(M_{1}-\left(Y_{1} \cup \ldots \cup Y_{u}\right)\right)$ satisfies the criteria for the 3-manifold $M$ of Lemma 1 . Hence, each is a handlebody.

By an application of Lemma 3 to the union of the essences $Y_{1}, \ldots, Y_{u}$ and to $\operatorname{cl}\left(M_{1}-\left(Y_{1} \cup \ldots \cup Y_{u}\right)\right)$, one now obtains a family of handlebodies, whose union will be called $T^{\prime}$, and one obtains for each $j=1, \ldots, u$ a submanifold $Y_{j}^{\prime}$ of $Y_{j}$ which is homeomorphic to $Y_{j}$ and which satisfies the other properties specified in the proof of Lemma 3.

(3.3) $T^{\prime}$ is a handlebody (i.e. $T^{\prime}$ is connected).

Proof of (3.3). Otherwise, the boundary of $M_{1}\left(=\mathrm{bd}\left(T^{\prime} \cup Y_{1}^{\prime} \cup \cdots \cup Y_{u}^{\prime}\right)\right)$ would not be connected. (See condition (iv) in the conclusion of Lemma 3.)

(3.4) For $j=1, \ldots, u, Y_{j}^{\prime} \cap T^{\prime}$ is the union of a family of disjoint disks, one lying on each component of bd $\left(Y_{j}^{\prime}\right)$.

Proof of (3.4). This follows from conditions (iii) and (iv) of the conclusion of Lemma 3.

Now let $F_{1}, \ldots, F_{u}$ be disjoint disks on bd $\left(T^{\prime}\right)$ such that for $j=1, \ldots, u$, $Y_{j}^{\prime} \cap T^{\prime}$ lies in the interior of $F_{j}$. And let $B_{1}, \ldots, B_{u}$ be disjoint 3-cells in the handlebody $T^{\prime}$ such that for $j=1, \ldots, u, B_{j} \cap$ bd $\left(T^{\prime}\right)=F_{j}$. Define

$$
T^{\prime \prime}=\operatorname{cl}\left(T^{\prime}-\left(B_{1} \cup \cdots \cup B_{u}\right)\right) .
$$

(3.5) For $j=1, \ldots, u, Y_{j}^{\prime} \cup B_{j} \approx P_{j}$.

Proof of (3.5). This follows immediately from statement (3.4) and Lemma 6.

(3.6) $M_{1} \approx P_{1} \triangle \cdots \triangle P_{u} \triangle T^{\prime \prime}$.

Proof of (3.6). By the construction in the paragraph preceding statement (3.5), $M_{1} \approx\left(Y_{1}^{\prime} \cup B_{1}\right) \triangle \cdots \triangle\left(Y_{u}^{\prime} \cup B_{u}\right) \triangle T^{\prime \prime}$. This decomposition of $M_{1}$ and statement (3.5) yield statement (3.6) immediately.

Correspondingly, the following statement holds:

(3.7) The 3-manifold $M_{2}$ is homeomorphic to the disk sum of $P_{u+1} \triangle \cdots \triangle P_{s}$ and a (possibly trivial) handlebody $U^{\prime \prime}$.

It follows from the Grushko-Neumann theorem (see Kurosh [2, p. 58]) that the sum of genus $\left(T^{\prime \prime}\right)$ and genus $\left(U^{\prime \prime}\right)$ is $n-s$. Therefore, statement A holds. 
4. Proof of the Decomposition Theorem. Let $N$ be a 3-manifold with vacuous boundary. In this section, $N^{*}$ denotes the 3-manifold obtained by removing from $N$ the interior of a 3-cell.

LEMMA 8. Let $M$ be a 3-manifold with connected, nonvacuous boundary, and let $N$ be a 3-manifold with vacuous boundary. Then $M \# N \approx M \triangle N^{*}$.

Proof. Let $E$ be the disk in $M \triangle N^{*}$ across which $M$ and $N^{*}$ are pasted, and let $B$ be the intersection of $N^{*}$ and the star of the disk bd $\left(N^{*}\right)-$ int $(E)$ in the second barycentric subdivision of $M \triangle N^{*}$. The 3-manifold obtained by removing $\mathrm{cl}\left(N^{*}-B\right)$ from $M \triangle N^{*}$ and filling in a 3-cell is homeomorphic to $M$, and $\operatorname{cl}\left(N^{*}-B\right)$ is homeomorphic to $N^{*}$. Therefore $M \triangle N^{*} \approx M \# N$.

Lemma 9. Let $M$ and $N$ be 3-manifolds in the class PCC. Then the disk sum $M \triangle N$ is also in the class PCC.

Proof. It is obvious that bd $(M \triangle N)$ is nonvacuous and connected. It will be shown that every 2-sphere in $M \triangle N$ bounds a 3-cell. Let $D$ be the disk in $M \triangle N$ across which $M$ and $N$ are pasted, and let $S$ be a 2-sphere in $M \triangle N$. A general position argument indicates that it suffices to consider the case in which each component of $S \cap D$ is a simple loop in the interior of $D$ at an actual crossing of the surfaces.

Basis step. If $S \cap D$ is empty, then $S$ lies either entirely in $M$ or entirely in $N$, in which case $S$ bounds a 3-cell in $M$ or $N$ respectively, because the 3-manifolds $M$ and $N$ are in the class $P C C$.

Induction step. Let $S \cap D$ have $n$ components, $n>0$. There is a component $k$ of $S \cap D$ which bounds a disk $R$ on the 2-sphere $S$ such that $R \cap D=k$. Let $E$ be the subdisk of $D$ which the loop $k$ bounds. For definiteness, one supposes that the disk $R$ lies in the 3-manifold $M$. Let $U$ be a neighborhood in $M$ of the disk $R$ and let $g: R \times[-1,1] \rightarrow U$ be a homeomorphism such that the following conditions hold:

(i) For all points $x$ in $R, g(x, 0)=x$.

(ii) $U \cap$ bd $(M)=g(k \times[-1,1]) \subset$ int $(D)$.

(iii) $g(k \times\{-1\}) \subset E$.

The 2-sphere which is the union of the disks $E \cup g(k \times[0,1])$ and $g(R \times\{1\})$ lies in the 3-manifold $M$, and since $M$ is the class $P C C$, it must bound a 3-cell in $M$. It follows that if $D^{\prime}$ denotes the disk $(D-(E \cup g(k \times[0,1])) \cup g(R \times\{1\})$, there is a homeomorphism $(M \triangle N, D) \rightarrow\left(M \triangle N, D^{\prime}\right)$. Since the number of components of $S \cap D^{\prime}$ is fewer than $n$, the 2-sphere $S$ bounds a 3-cell in $M \triangle N$. Hence, $M \triangle N$ is in the class $P C C$.

Proof of the Decomposition Theorem. Let $M$ be any 3-manifold with connected nonvacuous boundary. By Theorem 1, $M$ is homeomorphic to the connected sum of a 3-manifold $P$ in the class $P C C$ and a 3-manifold $Q$ with vacuous boundary. 
Let $P \approx P_{1} \triangle \cdots \triangle P_{r}$ be a $\triangle$-prime decomposition of $P$, and let $Q \approx Q_{1} \# \cdots \# Q_{s}$ be a \#-prime decomposition of $Q$. Then

$$
\begin{aligned}
M & \approx P \# Q \approx\left(P \# Q_{1}\right) \#\left(Q_{2} \# \cdots \# Q_{s}\right) \\
& \approx\left(P \triangle Q_{1}^{*}\right) \#\left(Q_{2} \# \cdots \# Q_{s}\right) \approx \cdots \approx P \triangle Q_{1}^{*} \triangle \cdots \triangle Q_{s}^{*},
\end{aligned}
$$

by Lemma 8 . Therefore, $M \approx P_{1} \triangle \cdots \triangle P_{r} \triangle Q_{1}^{*} \triangle \cdots \triangle Q_{s}^{*}$, which is a $\triangle$ prime decomposition of $M$.

Let $M \approx N_{1} \triangle \cdots \triangle N_{t}$ be another $\triangle$-prime decomposition of $M$, ordered so that $N_{u+1}, \ldots, N_{t}$ are the summands whose boundaries are 2-spheres. By Lemina 9, the 3-manifold $N_{1} \triangle \cdots \triangle N_{u}$ is in the class $P C C$. For $j=1, \ldots, t-u$, let $V_{j}$ be a 3-manifold with vacuous boundary such that $V_{j}^{*}=N_{j+u}$. The 3-manifold $V_{1} \# \cdots \# V_{t-u}$ has vacuous boundary, and the 3-manifold $M$ is homeomorphic to the connected sum of $N_{1} \triangle \cdots \triangle N_{u}$ and $V_{1} \triangle \cdots \triangle V_{t-u}$. By Theorem 1,

$$
N_{1} \triangle \cdots \triangle N_{u} \approx P_{1} \triangle \cdots \triangle P_{r} \text { and } Q_{1} \# \cdots \# Q_{s} \approx V_{1} \# \cdots \# V_{t-u} .
$$

By Theorem 3, $u=r$ and there is a reindexing of the $N_{i}$ 's such that for $i=1, \ldots, r$, $N_{i} \approx P_{i}$. By Theorem 2, $s=t-u$ and there is a reindexing of the $V_{i}$ 's such that for $i=1, \ldots, t=u, V_{i} \approx Q_{i}$, and, therefore, $V_{i}^{*} \approx Q_{i}^{*}$. The proof of the Decomposition Theorem is now complete.

\section{REFERENCES}

1. J. L. Gross, The decomposition of 3-manifolds with several boundary components (to appear).

2. A. G. Kurosh, The theory of groups. Vol. II, Chelsea, New York, 1956.

3. J. Milnor, A unique decomposition theorem for 3-manifolds, Amer. J. Math. 84 (1962), 1-7.

Princeton University,

Princeton, NeW Jersey 